\title{
Densification effects on the Boson peak in vitreous silica: a molecular-dynamics study
}

\author{
P. Jund and R. Jullien \\ Laboratoire des Verres, CNRS-Université Montpellier 2, \\ Pl. E. Bataillon CC069, 34095 Montpellier, France
}

We perform classical molecular-dynamics simulation to study the effect of densification on the vibrational spectrum of a model silica glass. We concentrate this study on the socalled Boson peak and compare our results, obtained from a direct diagonalization of the dynamical matrix, with experimental Raman data. We show that, upon densification, the position of the Boson peak shifts towards higher frequencies while its magnitude decreases which is in agreement with a recent experimental study.

PACS numbers: 61.43.Fs, 63.50.+x, 02.70.Ns

\section{INTRODUCTION}

Vitreous silica, the prototype material for the study of network forming glasses, displays a number of anomalous behaviors both in the structural and vibrational characteristics. This is also true when this material is exposed to high pressures. Experiments have shown that at room temperature glass samples compressed beyond $\approx 12 \mathrm{GPa}$ exhibit a permanent density increase of about $20 \%$. Both experiments $\theta^{-}$and numerical simulations have shown that this irreversible densification is mainly due to an increase of the average coordination number around the silicon atoms. An other topic concerns the influence of the pressure on the vibrational spectrum of vitreous silica. Very recently a substantial amount of experimental work has been dedicated to the study of this influence and more precisely to the effect of the densification on the so-called Boson peak (BP) 19 . The BP refers to an excess in the vibrational density of states with respect to the Debye distribution and is located generally around $1.5 \mathrm{THz}$. It is not clear yet if the BP only shifts towards higher frequencies upon densification or if this shift is a result of the suppression of the $\mathrm{BP}$. With the use of numerical simulations using a relatively realistic interaction potential one should be able to give a clear answer and connect this answer to the structural transformations observed in the afore mentioned studies 4 . 6 . As far as we know no simulations were done on the variation of the BP with the density. Here we present such a study in a model silica glass using the same pair-potential than Tse et al $\mathbf{6}$, the "BKS" potentiale. This potential has also been used recently to study pressure induced amorphization of quartz 11 and seems appropriate to describe silica at high pressure. Our results showing a shift of the $\mathrm{BP}$ as the density increases between 2.2 and 2.67 $\mathrm{g} / \mathrm{cm}^{3}$ are in relatively good agreement with the experimental Raman data and indicate a disappearance of the BP upon densification. In parallel we find similar results as Tse et al. .6 concerning the structural changes around the silicon atoms which are therefore not shown again here.

\section{SIMULATIONS}

We perform classical (constant energy, constant volume) molecular-dynamics simulations on an assembly of 648 particles $\left(216 \mathrm{SiO}_{2}\right.$ molecules) packed in a cubic box with periodic boundary conditions. The size of the box is fixed in order to study samples at the following densities: $2.17,2.32,2.40$ and $2.67 \mathrm{~g} / \mathrm{cm}^{3}$. The particles interact via the BKS potential1 10 with the same parameters as in our previous studies 12 . The low temperature glassy samples at a given density are obtained after a quench from the liquid state $(T \approx 7000 \mathrm{~K})$ at a constant quenching rate of $2.3 \times 10^{14} \mathrm{~K} / \mathrm{s}$. It is worth noticing that this procedure is different from the one used in 6 where a compression at room temperature was used in order to obtain the high density samples. After the quench, the samples at zero temperature are relaxed during 120000 timesteps (84 ps) (during which the temperature rises only slightly) and finally the vibrational spectrum $g(\nu)$ is obtained by direct diagonalization of the dynamical matrix. This direct diagonalization is the most computer time consuming since one has to deal with a $1944 \times 1944$ matrix with no a priori symmetry. Moreover since the Coulomb interactions have not been cut off, this matrix can not be considered as sparse (to reduce the computer time it should also be noted that we did not calculate the eigenvectors in this simulation). The eigenvalues of the dynamical matrix can also be obtained from the Fourier transform of the velocity-velocity autocorrelation function but in the low frequency region of the spectrum (which is the one we are mostly interested in) this technique is generally less reliable. For each density two independent liquid samples were used in order to improve slightly the statistics of the results. Because of the finite size of the simulation box we have discrete values of the frequency $\nu$ which are located between $\approx 0.8$ and $40 \mathrm{THz}$ in agreement with a previous study on the same system 13 . Nevertheless since we are interested in the low frequency part of the spectrum we will concentrate here on the frequency region below $10 \mathrm{THz}$. 


\section{RESULTS}

A standard way of extracting the BP from the vibrational spectrum is to plot $g(\nu) / \nu^{2}$ since in the Debye approximation $g(\nu) \approx \nu^{2}$ at low frequency. In fig.1 we show the averaged (over 2 samples) spectrum at different densities together with the neutron diffraction results 9 when available (the curves have been arbitrarily shifted for clarity). The solid lines in the figure are the fits of $g(\nu) / \nu^{2}$ by the following "generalized Lorentzian" functional form 14 :

$$
f(\nu)=f_{0} \cdot \nu^{n} \cdot \frac{1}{\left[\nu^{2}+\nu_{0}^{2}\right]^{m}}
$$

where $f_{0}, \nu_{0}, m$ and $n$ are adjustable parameters (this functional form is useful to describe the BP even though it has no direct physical meaning). In this figure the excess of modes between 1 and $2 \mathrm{THz}$ is clearly visible. Moreover a broadening of the peak when increasing the density can be seen in agreement with the experimental results. The increase of the peak position with increasing density is less pronounced than in experiment because of finite size effects. Indeed due to the limited size of our simulation box the number of frequencies "available" on the low frequency side of the spectrum is small. This explains the overestimated peak position at $2.2 \mathrm{~g} / \mathrm{cm}^{3}$ compared to the experimental data as has been shown recently 15 . We did the calculation for one sample at $2.2 \mathrm{~g} / \mathrm{cm}^{3}$ containing 1536 particles and obtained very similar results except a small shift of the peak position towards lower frequencies. Increasing the number of particles by a factor 2 improves barely the description of the peak, since the lower cut-off frequency is proportional to the inverse of the box size. In any case increasing the system size leads to a decrease of the peak position and an increase of its intensity (see 15 ) while increasing the density leads to an increase of the peak position and a decrease of its intensity as can be seen in fig. 1. The finite size effects are certainly responsible for the differences between the simulation and the experimental data but do not affect the general behavior with density. Since only 2 densities have been considered in the neutron diffraction study, we decided to compare off results to the more numerous Raman data available 8 . When doing this one has to be careful in order to compare things that are comparable. The reduced Raman intensity $I_{R}$ is related to the vibrational density $g(\nu)$ via a frequency-dependent function $C(\nu)$. Previous experimental studies 16 and a re cent study combining experiments and MD simulations 17 have indicated that $C(\nu) \approx \nu$ in the region of the $\mathrm{BP}$ which implies that $I_{R} \propto g(\nu) / \nu$. Hence in the following we use the function $g(\nu) / \nu$ and compare its characteristics to the available Raman data. To put this comparison on more quantitative grounds we fit the curves $g(\nu) / \nu=f(\nu)$ by a functional form similar to the one given in Eq. 1, and we extract the position of the maximum $\nu_{\max }$ as well as the intensity of the maximum $I_{\max }$ from the fits. In fig. $2 \mathrm{a}$ and $2 \mathrm{~b}$ the values of $\nu_{\max }$ and $I_{\max }$ are plotted as a function of the density respectively and compared to experimental Raman values. In fig.2a, the 2 points reproduced from (open squares) which is a study of the vibrational properties of silica as a function of pressure were obtained assuming a $15 \%$ densification of the high pressure sample as stated by the authors.

\section{DISCUSSION}

The results reported in fig. 2 show clearly that the BP is shifted towards higher frequencies and its intensity vanishes when the density of our model silica glass increases. In fact, compared to fig.1, not surprisingly, the relative variations of the peak position and intensity are more important when $g(\nu) / \nu$ is used. In fig.2a we see that the overall trend of the BP position as a function of density is correct even though our MD simulations overestimate this position compared to the experimental data which is probably due to finite size effects as stated earlier. Nevertheless it appears that our results come closer to the experimental data at high density even though the positive curvature seems to be absent in the simulation (the error bars in fig. $2 \mathrm{a}$ and $2 \mathrm{~b}$ result from the dispersion over the 2 samples studied here and therefore should be considered as a rough estimate of the error). This shows that the shift of the BP towards higher frequencies is more important than the artificial drift due to the fact that with increasing density (decreasing system size) the lower cut-off frequency increases in our simulations. This increase would imply a worse description of the BP region but since the BP shifts towards higher frequencies our description becomes (relatively) better at high density. Another point concerns the influence of the rapid quenching rate: indeed Vollmayr et al. have shown that the height and to a lesser-degree the shape of the BP depend on the cooling rate 18 . Nevertheless, similarly to the finite size effects, these authors show that a smaller cooling rate would lead to a behavior of the $\mathrm{BP}$ opposite to the one observed with increasing density. Therefore since all the samples have been quenched at the same rate the evolution of the BP with the density can not be linked to cooling rate effects. In fact all the previous studies show that using larger system sizes and smaller cooling rates would lead to a better agreement of the curves plotted in fig. 2 a especially at low density but would not affect the behavior of the BP with the density which is the aim of this study.

Differences can also be seen between the experimental studies. The principal distinction between the two experimental procedures is the temperature since Sugai et al. performed the densification at room temperature while Inamura et al. compressed the samples at $700 \mathrm{C}$. This might explain why our low temperature simulations are closer to the experimental results obtained at room temperature. In order to check the effect of the temperature, 
we performed some calculations on samples quenched at $300 \mathrm{~K}$ but no major differences were noticeable. Also we tried a "cold compression" at 300K followed by a long relaxation run (200 ps) but again the spectra were similar to the ones reported in fig.1. The results in fig. $2 \mathrm{~b}$ confirm that with increasing density the BP tends to disappear. Again we do not find the almost linear decrease observed in experiment at low density but such a behavior can be observed for the higher densities (this shows again that our description becomes better at high densities). Since we fixed the calculated and experimental intensity of the BP to 1 at the usual density $\left(2.2 \mathrm{~g} / \mathrm{cm}^{3}\right)$ for which we know that the calculated intensity is underestimated because of the finite size of the simulation box, we have a systematic overestimation of the relative intensity of the BP at higher density. Nevertheless the overall trend is clear and it is coherent with the data of Inamura et al 9 who recently showed not only a shift towards high frequencies but also a disappearance of the BP. In their publication these authors attributed the suppression of the low-energy dynamics to the shrinkage of the void space by densification 19 . This analysis is coherent with the soft potential model (the soft modes in the void space have been suppressed by shrinkage) but seems to contradict the analysis of the shift of the BP being due to phonon scattering by local density fluctuations which would result in a shift of the BP onlyt. Nevertheless the microscopic model of the BP is not settled yet since the structural entities involved in the soft potential model have not been clearly identified. The connection between this study and the previous numerical studies investigating the structural changes under densification should permit to progress in such an identification.

\section{CONCLUSION}

We performed classical molecular-dynamics simulations combined with the diagonalization of the dynamical matrix to investigate the influence of the density on the Boson Peak in a model silica glass. Even though we used a relatively small system size and a rather fast cooling rate, the results compare relatively well with experimental Raman data and show that upon densification the Boson Peak shifts to higher frequencies while its intensity decreases. These results confirm the disappearance of the BP in the high density samples as shown in a recent experimental study which could be due to a shrinkage of the void space 19 . The quantitative agreement between our simulations and experiment is not perfect (it should be noted that differences exist also between the experimental data) but we know the reasons of this discrepancy. Nevertheless the BKS potential is able to give the correct behavior of the vibrational spectrum under compression and this shows once more the good quality of this pairwise interaction. Of course since we did not calculate the eigenvectors we have no informations on the nature of the modes dissapearing under compression. But the disappearance of the excess of low-energy modes under densification is the first step in the clear identification of what the vibrating structural entities are. To perform this identification in a simulation one needs tools more accurate than the radial pair distribution function or the Voronoï tessellation. Also if the connection between the $\mathrm{BP}$ and the plateau in the thermal conductivity exists one should see a variation of the plateau with the density. this variation has already been reported experimentallye but not yet confirmed in a numerical simulation.

\section{ACKNOWLEDGMENTS}

We would like to thank M. Foret and C. Levelut for helpful discussions on this work. Part of the simulations have been done at the Centre Informatique National de l' Enseignement Superieur (Montpellier).

${ }^{1}$ J. Zarzycki, Les verres et l'état vitreux (Masson, Paris, 1982).

${ }^{2}$ P.W. Bridgman and I. Simon, J. Appl. Phys. 24, 405 (1953).

3 J.D. Mackenzie, J. Am. Ceram. Soc. 46, 461 (1963); G.E. Walrafen and P.N. Krishnan, J. Chem. Phys. 74, 5328 (1981); M. Grimsditch, Phys. Rev. Lett. 52, 2379 (1984); P. Mc Millan, B. Piriou and R. Couti, J. Chem. Phys. 81, 4235 (1984); R.J. Hemley, H.K. Mao, P.M. Bell and B.O. Mysen, Phys. Rev. Lett. 57, 747 (1986); R.A.B. Devine, R. Dupree, I. Farnan and J.J. Capponi, Phys. Rev. B 35, 2560 (1987); R.A. Murray and W.Y. Ching, Phys. Rev. B 39, 1320 (1989); S. Susman, K.J. Volin, R.C. Liebermann, G.D. Gwanmesia and Y. Wang, Phys. Chem Glasses 31, 144 (1990); C. Meade, R.J. Hemley and H.K. Mao, Phys. Rev. Lett. 69, 1387 (1992); A. Polian and M. Grimsditch, Phys. Rev. B 41, 6086 (1990); C.S. Zha, R.J. Hemley, H.K. Mao, T.S. Duffy and C. Meade, Phys. Rev. B 50, 13105 (1994).

${ }^{4}$ R.A.B Devine, R. Dupree, I. Farnan and J.J. Capponi, Phys. Rev. B 35, 2560 (1987); R.A.B. Devine and J. Arndt, Phys. Rev. B 35, 9376 (1987); Q. Williams Q. and R. Jeanloz, Science 240, 902 (1988); S. Susman, K.J. Volin, D.L. Price, M. Grimsditch, J.P. Rino, R.K. Kalia, P. Vashishta, G.D. Gwanmesia, Y. Wang and R.C. Liebermann, Phys. Rev. B 43, 1194 (1991).

${ }^{5}$ L. Stixrude and M.S.T. Bukowinski, Phys. Rev. B 44, 2523 (1991); J. Wei, R.K. Kalia, P. Vashishta and J.P. Rino, Phys. Rev. B 50, 118 (1994); R.G. Della Valle and E. Venuti, Phys. Rev. B 54, 3809 (1996).

${ }^{6}$ J.S. Tse, D.D. Klug and Y. Le Page, Phys. Rev. B 46, 5933 (1992).

${ }^{7}$ S. Sugai and A. Onodera, Phys. Rev. Lett. 77, 4210 (1996); 
S. Sugai, H. Sotokawa, D. Kyokane and A. Onodera, Physica B 219-220, 293 (1996).

${ }^{8}$ Y. Inamura, M. Arai, N. Kitamura, S.M. Bennington and A.C. Hannon, Physica B 241-243, 903 (1998).

${ }^{9}$ Y. Inamura, M. Arai, O. Yamamuro, A. Inaba, N. Kitamura, T. Otomo, T. Matsuo, S.M. Bennington and A.C. Hannon, Physica B 263-264, 299 (1999).

${ }^{10}$ B.W.H. van Beest, G.F. Kramer and R.A. van Santen, Phys. Rev. Lett. 64, 1955 (1990).

${ }^{11}$ J. Badro, P. Gillet and J.L. Barrat, Europhys. Lett. 42, 643 (1998).

12 P. Jund and R. Jullien, Phys. Rev. B 59, 13707 (1999); P. Jund and R. Jullien, Phil. Mag. A 79, 223 (1999).

13 S.N. Taraskin and S.R. Elliott, Phys. Rev. B 55, 1 (1997).

${ }^{14}$ F. Terki, C. Levelut, J.L Prat, M. Boissier and J. Pelous, J. Phys: C.M. 9, 3955 (1997).

${ }^{15}$ J. Horbach, W. Kob and K. Binder, cond-mat/9910445

${ }^{16}$ A.P. Sokolov, U. Buchenau, W. Steffen, B. Frick B. and A. Wischnewski, Phys. Rev. B 52, R9815 (1995); A.P. Sokolov, Physica B 219-220, 251 (1996).

17 A. Fontana, R. Dell'Anna, M. Montagna, F. Rossi, G. Viliani, G. Ruocco, M. Sampoli, U. Buchenau and A. Wischnewski, Europhys. Lett. 47, 56 (1999).

${ }^{18}$ K. Vollmayr, W. Kob and K. Binder, Phys. Rev. B 54, 15808 (1996).

19 S.R. Elliott, Phys. Rev. Lett. 67, 711 (1991).

${ }^{20}$ D.M. Zhu, Phys. Rev. B 50, 6053 (1994).

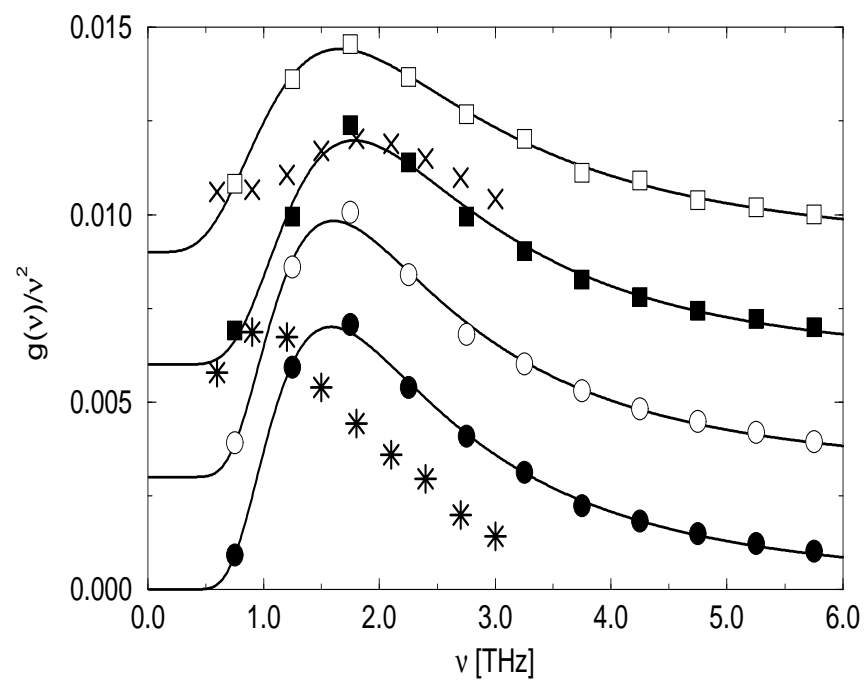

FIG. 1. Plot of $g(\nu) / \nu^{2}$ versus $\nu$ as a function of density: •: $2.17 \mathrm{~g} / \mathrm{cm}^{3}$; ○: $2.32 \mathrm{~g} / \mathrm{cm}^{3}$; $\mathbf{\square}: 2.40 \mathrm{~g} / \mathrm{cm}^{3} ; \square: 2.67 \mathrm{~g} . \mathrm{cm}^{3}$. Comparison with experimental neutron diffraction datal (the intensity at $2.2 \mathrm{~g} / \mathrm{cm}^{3}$ has been adjusted to the corresponding MD intensity ): $*: 2.2 \mathrm{~g} / \mathrm{cm}^{3} ; \times: 2.63 \mathrm{~g} / \mathrm{cm}^{3}$.

The solid lines correspond to the fit of the MD results with the "generalized Lorentzian" given in Eq. 1 (see text)
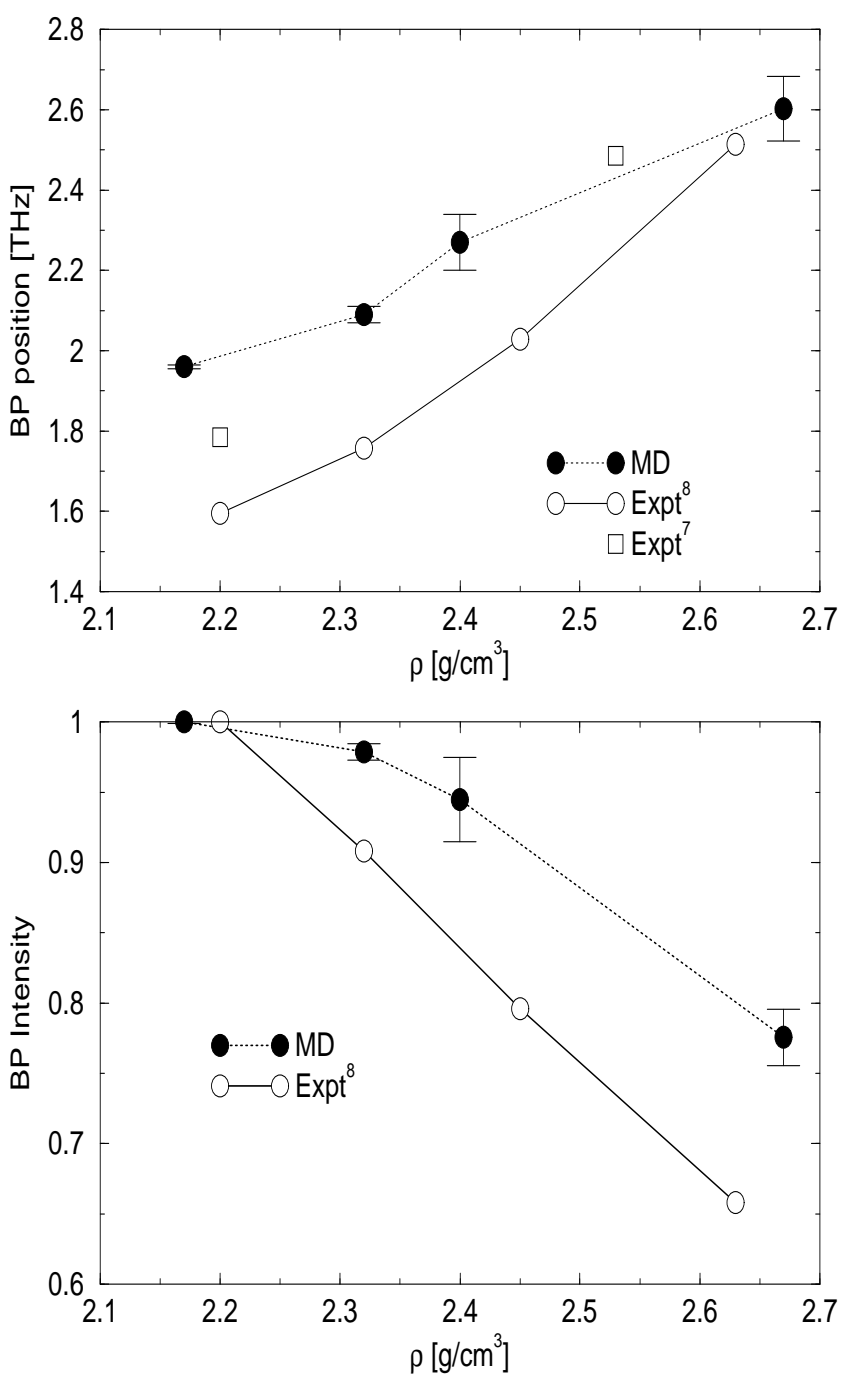

FIG. 2. (a) Variation of the position of the BP as a function of density; (b) Variation of the intensity of the BP as a function of density. 